Paradise Sold: Resource Partitioning and the Organic Movement in the US Farming Industry

Organization Studies 34(5-6) 623-65I

(c) The Author(s) 2013

Reprints and permissions: sagepub.co.uk/journalsPermissions.nav DOI: 10.1 177/01708406/3479225 www.egosnet.org/os

(SAGE

\title{
Katarina Sikavica
}

Ludwig Maximilians University Munich, Germany

\section{Jo-Ellen Pozner}

University of California Berkeley, USA

\begin{abstract}
Resource partitioning theory maintains that in markets in which anti-mass-production cultural sentiments make producer identity relevant, there should be no direct competition between generalists and specialists. Nevertheless, anecdotal evidence suggests that after initial partitioning, such competition, and hence departitioning, is in some cases possible. We refine received insights of resource partitioning theory regarding the stability of niche markets, particularly those driven by identity movements, by introducing the notion that partitioning is a dynamic and even reversible process. Previous research has offered an answer to the question of why identity movements create partitioning: because they increase the dimensionality of the resource space and engender sanctioning of visible violations of the specialists' organizational form identity. In contrast, we offer an answer to the question of how and when markets may partition in a stable way: by generating sharply defined specialist form identities whose definitional code includes limits to organizational growth. Identity movements are underpinned with mechanisms that can facilitate or inhibit market partitioning, depending on their ability to generate sharp specialist form identities. We illustrate our argument through the case of organic farming in the United States, with reference to prior work on microbrews and micro-radio, and discuss implications for resource partitioning theory.
\end{abstract}

\section{Keywords}

identity movements, organic farming, resource partitioning

*Both authors contributed equally to this paper.

Corresponding author:

Katarina Sikavica, Institute of Strategic Management, Ludwig Maximilians University, Ludwigstrasse 28 RgB, 80539

Munich, Germany.

Email: sikavica@bwl.Imu.de 
Resource partitioning theory provides a useful lens for investigating market structure and the viability of small, specialist organizations. Resource partitioning describes a market equilibrium where generalist and specialist organizations occupy distinct market niches so as not to tap the same resource space or enter into direct competition (Carroll, 1985; Carroll, Dobrev, \& Swaminathan, 2002). Previous research portrays resource partitioning as a dichotomous and definitive outcome: markets are considered as either partitioned or not. Once partitioned, they are assumed to remain stable without much overlap between generalists' and specialists' niches. Although this theory is comprehensive with respect to the end state of market structure and partitioning, our research suggests that refinement is needed to account for market dynamics and specialists' viability over time.

Refinement is particularly appropriate for markets in which social movements, typically fuelled by anti-mass-production cultural sentiments, form the basis for partitioning. Movements are inherently dynamic phenomena, unfolding in stages: they emerge, grow, and eventually decline. Resource partitioning theorists reason that social and identity movements enhance specialists' viability by increasing the dimensionality of the resource space and by creating resource pockets for specialists to exploit (e.g., Carroll \& Swaminathan, 2000; Greve, Pozner, \& Rao, 2006; Soule \& King, 2008). In the micro-brewing and micro-radio cases, for example, strong identity movements are found to be important drivers of partitioning and are identified as successful triggers of specialist proliferation. Nevertheless, the organic farming case indicates that this is not always true; after initial partitioning, the market for organic produce became much more unstable and unstructured than the micro-radio and micro-brew markets. Perhaps even more importantly, despite the movement's success and an ever-growing number of followers, conventional farmers were able to enter the organic space such that organic farmers were not shielded from direct competition with them, threatening the economic viability of the specialist organic farmers. It follows that while extant research provides the reason why movements induce partitioning, it is not unequivocally clear how and when this will be the case.

To better understand the conditions underlying durable partitioning and stable market niches, we take seriously the dynamic nature of movements and partitioning. We juxtapose the microbrew, micro-radio, and organic farming movements and seek to understand the similarities and differences between the three. We adopt a longitudinal view using "thick" qualitative data to reconstruct the organic farming movement as a historical case study, and engage in what Ragin (1994) termed "reciprocal clarification," using extant research on the micro-radio and micro-brew movements (Carroll \& Swaminathan, 2000; Greve et al., 2006) to identify the key mechanisms that drive partitioning. We then compare and reconcile the similarities and differences between the mechanisms underlying previously studied movements and those underpinning the organic farming movement as it unfolded.

In line with previous research, we base our investigation on the assumption that market partitioning largely depends on the establishment of a specialist organizational form identity that is sufficiently narrow and sharply defined to sustain the boundaries between generalist and specialist organizations (McKendrick \& Carroll, 2001). Narrow and sharply defined specialist form identities are important because they provide fertile ground for movement participants' identification with specialist organizations, which is necessary for the detection and sanctioning of visible form violations (Foreman \& Whetten, 2002). We find that identity movements differ with respect to several mechanisms that drive organizational form identity: goal formation; framing and discourse; mobilization; and the nurturing of a supportive social infrastructure. We show that, despite initial market partitioning, the failure of the organic farming movement to generate a 
sharply defined specialist form identity rendered identification with specialists and sanctioning of specialist form violations difficult, and resulted in market de-partitioning, and ultimately repartitioning. Our analysis leads us to conclude that, beyond oppositional identity, a sharply and narrowly defined specialist form identity must include small-scale production and limits to organizational growth to generate stable partitioning; without these implicit limits to growth, a more complex and fractured market is likely to emerge. Our investigation thus challenges received insights of resource partitioning theory regarding the size and stability of niche markets by portraying resource partitioning in the presence of identity movements as a more dynamic, multifaceted, and potentially reversible process.

We proceed as follows. We first elaborate on our data and methods. Subsequently, we provide a brief historical narrative of the organic farming movement. We then use prior work on the microbrew and micro-radio movements to identify the mechanisms underlying stable resource partitioning and compare the similarities and differences with the organic farming movements. We close by offering a re-specification of the original resource partitioning model before moving to alternative explanations and a discussion of our results.

\section{Research Design and Methods}

Most research on resource partitioning adopts a quantitative approach, in the form of retrospective data collection and analysis where the factors that induced partitioning and influenced the shape of markets are analyzed post hoc, i.e., after the market structure has been identified as partitioned. However, as McKendrick and Carroll (2001) note, while such an approach may be very insightful for understanding what occurred, it is limited in its ability to explain why it occurred and when it is most likely to happen. In particular, there is a danger that the independent variables that are identified a posteriori in extant research on resource partitioning may be either spurious or insufficient for producing a partitioned market (McKendrick \& Carroll, 2001). An additional difficulty with the quantitative statistical approach in studies investigating movements as triggers of partitioning is the dynamic and process-like nature of social movements: because movements unfold in stages it seems reasonable to assume that the factors pertinent to partitioning may change over the course of the movement, leading to path dependencies that may impede or enhance the likelihood of partitioning. As a result, the factors necessary and sufficient to induce partitioning may be even more difficult to detect, and a methodological approach that accounts for the embedded and temporally interconnected nature of movement stages seems indicated.

To overcome these "thorny inference problems" (McKendrick \& Carroll, 2001, p. 665) we adopt a qualitative, case-based approach. We follow McKendrick and Carroll's (2001) interpretation of Ragin's (1994) approach and use a method described as "reciprocal clarification," a process that "clarifies concepts (the key analytical frames) and empirical categories in a reciprocal manner" (Ragin 1994, p. 88). In doing so, we assemble data on the organic movement and market structure of US farms over time and juxtapose this data to what we know from extant research at the intersection between social movements and resource partitioning theory - our key analytical frames or theoretical orienting ideas.

Like much qualitative research our approach is somewhat circular; knowledgeable about the premises of resource partitioning theory we started out with an empirical observation: examining the market structure of US farms, we were surprised to learn that despite the organic movement's success, the market for farming products in its present state cannot be described as partitioned. This observation motivated us to examine more closely what research at the intersection between 
identity movements and resource partitioning had to say about the factors necessary and sufficient to induce partitioning and to use these analytical frames to inform our theory. Thus, the presence of a strong social movement gave us the opportunity to theorize about the role of identity movements in creating resource partitioning, thereby adding dynamism and refinement to the population ecology story. In doing so, much like McKendrick and Carroll (2001), we seek avoid the dominant retrospective analysis problem: because the organic movement is still at the peak of its popularity and the market for farming produce has not yet reached its end state, by observing what happens before its establishment we can learn more about the necessary and sufficient conditions generating it.

\section{Case Methodology}

Yin (1998, p. 18) defines a case study as an empirical inquiry that "investigates a contemporary phenomenon in depth and within its real-life context" which is particularly suitable when "the boundaries between phenomenon and context are not clearly evident." He further notes that the case-study inquiry is useful for dealing with the unfortunate situation of having more variables of interest than data; consequently, it incorporates evidence from multiple sources and relies on existing theory to guide data collection and analysis. According to Yin (1984), a case study thus is an all-encompassing method covering the logic of design, data collection, and data analysis. This was the approach we took in conducting our study: starting with a historical reconstruction of the organic farming movement in the USA and data about market structure, we used extant theory as well as research on the micro-brew and micro-radio movements to distill the mechanisms underlying resource partitioning. Subsequently, we compared the similarities and differences among the three movements to refine resource partitioning theory in terms of how and when identity movements will encourage partitioning.

In terms of data collection, we used multiple sources of evidence to triangulate data. To increase the validity of our study and corroborate the facts uncovered, we collected information from documentation, archival records, and interviews (see Yin, 1984). For the same purpose, we assured that multiple collectors (the authors and five research assistants) were engaged in gathering the data (investigator triangulation); and, to keep a good overview, we installed an Internet homepage where all investigators contributed to the case-study database and case-study protocol.

Moreover, we followed Ragin (1994) and McKendrick and Carroll (2001) and used both structured and unstructured methods of data collection. Our structured methods involved collection of as much quantitative data on the development of organic farming - which is sparse - as possible. The data available were collected in the 2007 Census of Agriculture, conducted by the National Agricultural Statistics Service of the US Department of Agriculture (USDA), as well as the Economic Research Service of the USDA, which in turn was collected by USDA-accredited state and private organic certifiers; although we have exploited these data to the fullest, the available statistics are quite limited. We also systematically reviewed the academic and popular literature on organic farming, including academic journals, books, and Internet searches. Our unstructured methodology involved interviewing and corresponding with experts in the organic farming movement and industry, including academics and representatives of non-profit agencies servicing organic farmers. All evidence collection was documented in our case-study protocol and stored in our case-study database for further analysis.

Our data analysis technique is best described by what Yin (1984) terms "pattern matching logic." According to the author, such a logic "compares an empirically based pattern with a predicted one." In our case, this translates into comparing the mechanisms that generate resource partitioning identified in extant research to the evidence from the organic farming movement. 
Because, as we note, movements are dynamic phenomena that unfold in stages that emerge, grow and decline, our data analysis also involved a longitudinal approach; we identified the stages of the organic farming movement, and investigated resource partitioning and market structure in each case separately.

We present our case in an inductive manner starting with a historic narrative of the organic farming movement as well as data on the farming market structure at multiple points in time. Subsequently, we elaborate on the mechanisms we identified underlaying identity movements before "unpacking our case" and reconciling our theoretical orienting ideas with our evidence.

\section{The Organic Farming Movement}

In this section, we present the history of the organic farming movement and the industry it created. Our focus is on basic agriculture - farm production of grains, fruits, and vegetables - leaving aside the manufacture and distribution of processed organic foods. A summary of the major developments in US agriculture and the organic farming movement can be found in Table 1.

\section{Movement Origins}

The roots of the organic farming movement in the USA reach back to the 19th century, just as the use of synthetic fertilizers to increase crop yields took hold. During the Second World War, activists such as Jerome Rodale popularized the idea of a holistic approach to "natural," local, sustainable farming. While there was never a clear definition of organic farming practice, most early organic farmers used organic material in place of chemical fertilizers and pesticides, integrated livestock into a symbiotic farm system, and rotated multiple crops rather than cultivating single crops (Concord, 2001; Fromartz, 2006; Heckman, 2006). The movement gained a small, devoted following, but it was not until its ideals were taken up by environmental activists and members of the anti-establishment movement in the 1960s and 1970s that the movement really took root. The "back-to-the-land" and environmental movements that arose in response to urbanization, hyperindustrialization, and consumerism found common cause with the organic movement's ideals (Vos, 2000). During this period, consumption of organic produce was limited to those devotees willing to pay a premium. In the 1940 s, the majority of organic produce was sold directly to the consumer, and could only be found in small, niche healthfood stores from the 1950s through the 1980s (Greene, 2001).

A turning point came during the farm crisis in the 1980s, when economizing conventional farms began to harvest crops without pesticides. Finding they could earn a premium, they entered the organic market for purely economic reasons (Sustainable Agriculture Research and Education, 2008 ) and encouraged other conventional farmers to enter the highly profitable organic sector. The result of the entry of these large-scale, quick-growing farms was a bifurcated industry comprising "agrarians" - small-scale, specialty farmers who viewed organic farming as an alternative to industrial farming - and "expansionists" - more business-minded, single-crop producers "without any of the early ideological baggage to hold them back" (Fromartz, 2006), interested in increasing scale and broadening the market.

\section{The Struggle to Define Organic}

As the organic movement grew, informal organizations defined and enforced standard practices at the local level. An early proponent of formal definition of standard practices was a certification program adopted by agrarian farmers, formalized as the California Certified Organic Farmers 


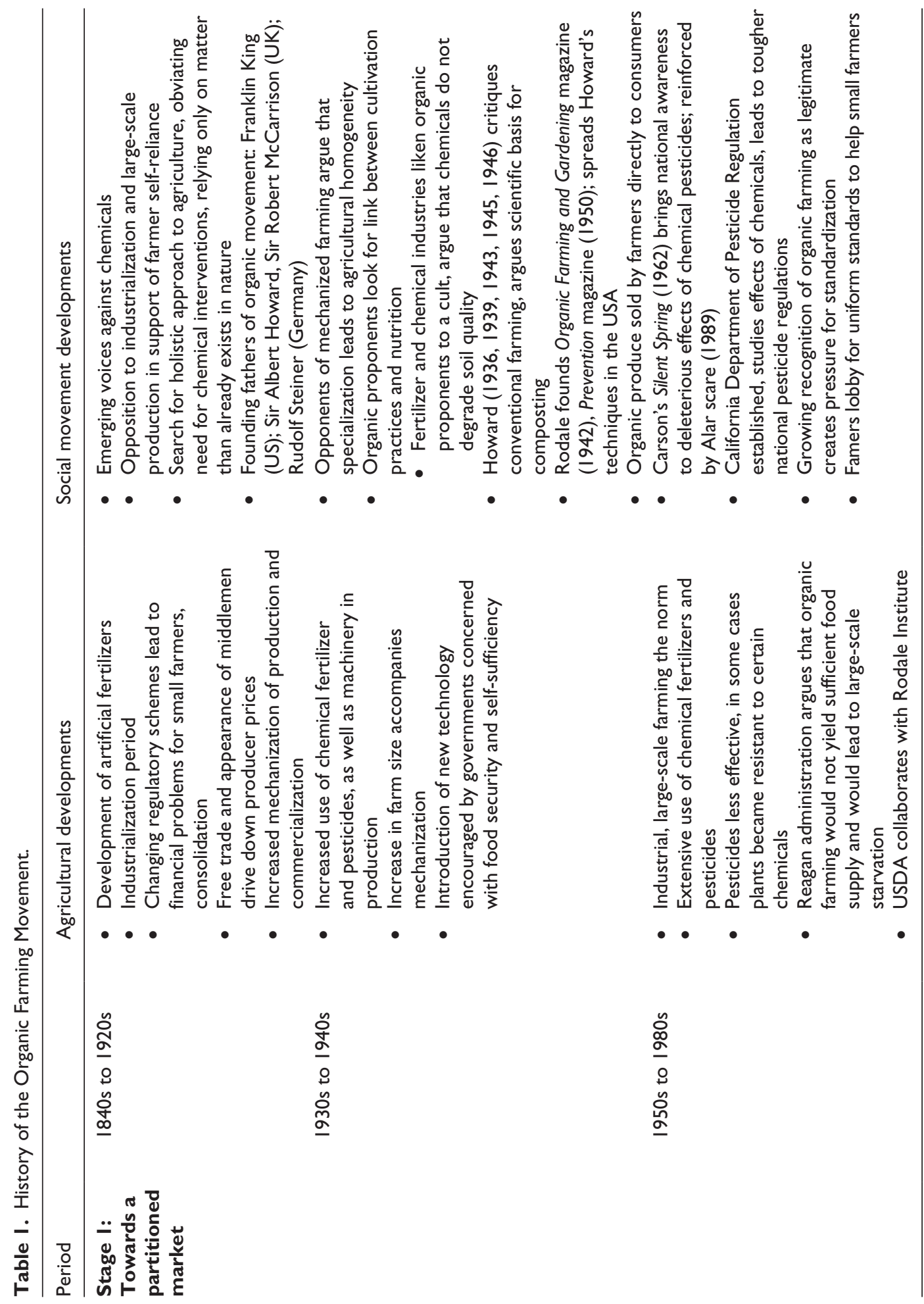




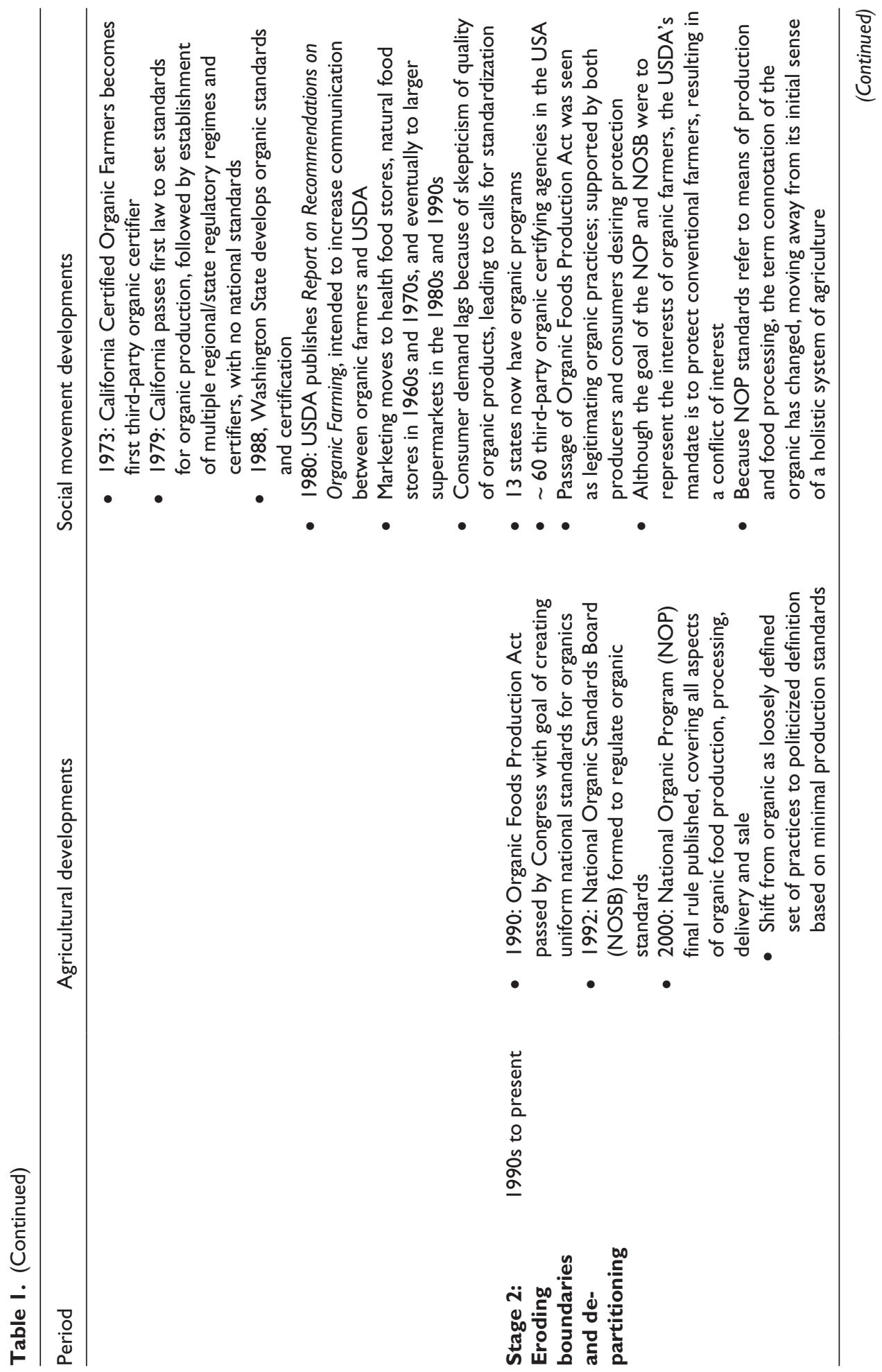




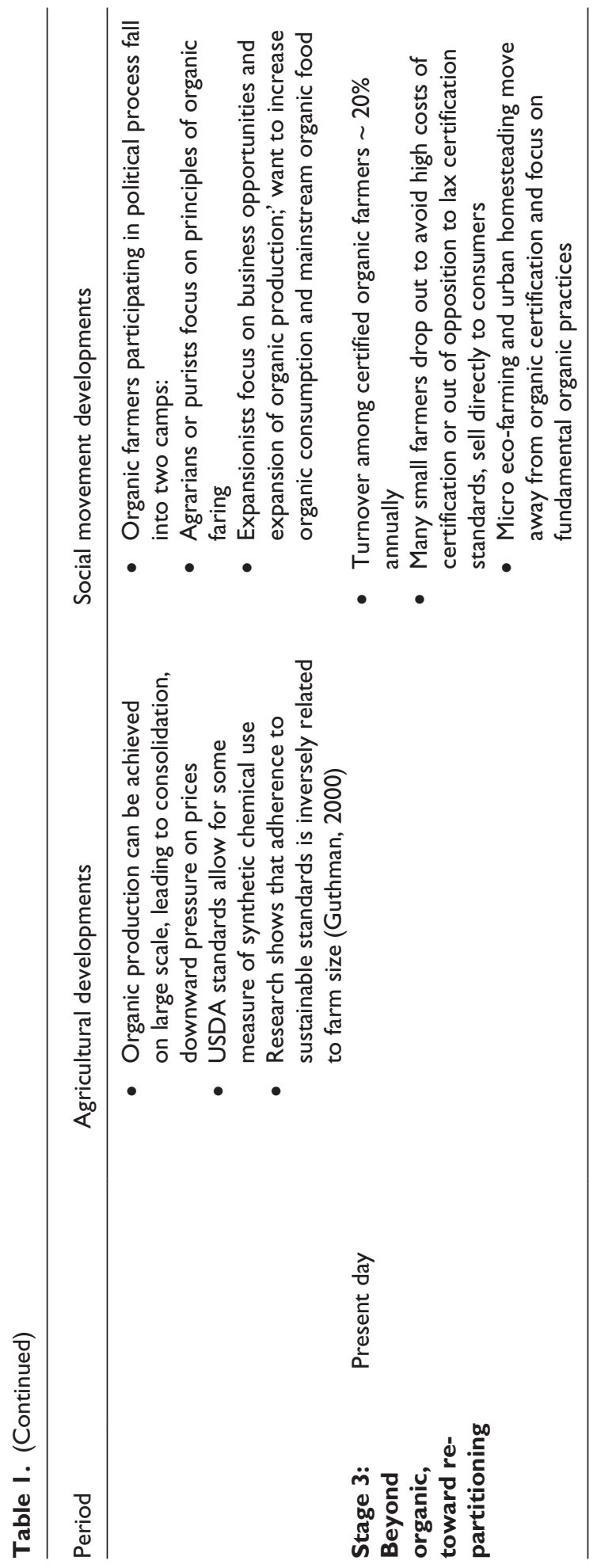


(CCOF) in 1973 (Guthman, 2004). Organizations like the CCOF typically consisted of independent farmers who favored standardization for the sake of consumer education and protection (Guthman, 2004). Because initial efforts were undertaken at the state or local levels, competing certification regimes emerged; the definition of organic farming thereby became subject to interpretation by the certifying agent, with some defining it as production using traditional organic methods employed by small, family farms in harmony with nature (King, 2007), while others emphasized sustainability, independence, and fair pricing (Fromartz, 2006), or environmental protection and repair (Ness, 2006), and still others were concerned with organic inputs rather than processes (Buck, Getz, \& Guthman, 1997).

The idea of federal certification gained currency following the Alar apple pesticide scare in 1989 and a number of high-profile organic scams (Fromartz, 2006; Guthman, 2004). The 1990 farm bill included the Organic Foods Production Act (OFPA), creating the National Organic Standards Board (NOSB) to oversee certification and determine the methods to be included in the national organic standards (Nestle, 2007). The NOSB immediately came to blows with the USDA, which was seen to represent the "big boys" of the conventional farming industry (Fromartz, 2006), partially due to the influence of other government agencies and interests on the USDA's regulatory process (Fromartz, 2006) and the fundamental conflict of interest between agrarians and expansionists (Buck et al., 1997; Guthman, 2004; Vos, 2000). Whereas organic farmers' greatest challenge previously came from conventional farmers, now the greatest conflict came from within organic circles, which comprised two conflicting visions of the meaning of organic farming (Vos, 2000). Pollan (2006, p. 155) describes this divisive regulatory effort as a struggle "between Big and Little Organic - or...between the organic industry and the organic movement...Big Organic won all [the] arguments."

\section{The Changing Organic Field}

Whereas early organic consumption was limited to movement adherents and health fanatics, today a much larger proportion of the public regularly consumes organic produce. Today the number of consumers eating organic has grown to the point that discerning members of the movement are outnumbered by casual consumers. Recent research shows that only one-third of organic purchasers are committed, zealous organic consumers, while the majority are pragmatic consumers who try to fit organics into their lifestyles (Natural Marketing Institute, 2005). Similarly, conventional markets overtook natural food stores as the primary marketer of organics in 2000 (Sligh \& Christman, 2003). Moreover, as new, expansionist producers entered the organic market, large corporations came to dominate, and there is evidence of consolidation among small organic farms due to the shrinking price premium for organic produce and the costs of maintaining organic certification, which disproportionally affects small farmers (Pollan, 2006). Consolidation down the value chain puts downward pressure on organic prices, creating pressure to exploit potential scale economies (King, 2007).

Whereas farms of agrarian movement adherents are generally small, the percentage of small farms with organic certification has decreased (Walz, 2004), while the number of larger farms has grown. As the total number of small farms has recently increased (see Table 2), this suggests that small farmers are either opting out of costly organic certification or are merging and consolidating into larger operations. Recent surveys show that, while gross sales acreage farmed organically in California has grown, the number of organic farms has fallen (Klonsky, Tourte, Kozloff, \& Shouse, 2002), indicating consolidation. At the other end of the spectrum, the number of large farms (over 2000 acres) has grown, primarily at the expense of mid-sized farms, suggesting concentration 
throughout the industry. The structure of the organic farming sector is growing to mirror that of conventional farming, with 3 percent of farms in each segment falling into the category of "very large farms" with over $\$ 500,000$ in sales (Lohr \& Park, 2002). This is also suggested by growth trends in certified organic farmland (see Table 3). According to the USDA, the change in certified organic acreage increased by 123 percent between 2002 and 2007, whereas the number of certified organic farms increased by only 55 percent (see Table 4 for details on certification); the clear inference is that recently certified organic farms are larger than earlier organic farms. Consolidation is distressing to agrarians, as farmers' ecological ratings tend to deteriorate as the scale of the farm increases (Guthman, 2000).

In response to organic farm consolidation, a small, dedicated band of agrarian-identified farmers recently launched the micro eco-farming movement. Micro eco-farms, defined as sustainable, local farms that range "from urban greenhouses to backyard gardens to small rural 1 to 25 -acre parcels" (Center for the Micro Eco-Farming Movement, 2010), are committed to "earth regenerating and socially just mini-farming." The movement ideology harkens back to the Rodale-inspired organic movement, with an emphasis on bio-dyanamic farming, natural fertilizers, and saving and promulgating endangered varieties of fruits and vegetables, and is oriented towards commercialization. The movement is gaining traction through alliance with other groups associated with local food, fair trade, seasonal eating, nature education and eco-tourism, and environmental sustainability causes. Movement adherents are uninterested in organic certification, which they view as not only costly but also too watered down to have meaning (Barnett, 2011).

Table 2. US Agricultural Land Use, 2002 and 2007.

\begin{tabular}{|c|c|c|c|c|}
\hline \multirow[t]{2}{*}{ All Farms } & \multicolumn{2}{|l|}{2002} & \multicolumn{2}{|l|}{2007} \\
\hline & Total & $\%$ Total & Total & $\%$ Total \\
\hline Farms (number) & $2,128,982$ & $100.0 \%$ & $2,204,792$ & $100.0 \%$ \\
\hline Farms (acres) & $938,279,056$ & $100.0 \%$ & $922,095,840$ & $100.0 \%$ \\
\hline Total cropland farms (number) & $1,751,450$ & $82.3 \%$ & $1,685,339$ & $76.4 \%$ \\
\hline Cropland farms (acres) & $434,164,946$ & $46.3 \%$ & $406,424,909$ & $44.1 \%$ \\
\hline Harvested cropland farms (number) & $1,362,608$ & $64.0 \%$ & $1,328,004$ & $60.2 \%$ \\
\hline Harvested cropland farms (acres) & $302,697,252$ & $32.3 \%$ & $309,607,601$ & $33.6 \%$ \\
\hline \multicolumn{5}{|l|}{ Farms by acres harvested: } \\
\hline I to 49 acres & 688,492 & $50.5 \%$ & 700,266 & $52.7 \%$ \\
\hline I to 9 acres & 223,038 & $16.4 \%$ & 233,119 & $17.6 \%$ \\
\hline 10 to 19 acres & 177,964 & $13.1 \%$ & $|82,5| 4$ & $13.7 \%$ \\
\hline 20 to 29 acres & $126,7 / 3$ & $9.3 \%$ & 126,330 & $9.5 \%$ \\
\hline 30 to 49 acres & 160,777 & $11.8 \%$ & 158,303 & $11.9 \%$ \\
\hline 50 to 99 acres & 186,980 & $13.7 \%$ & 179,055 & $13.5 \%$ \\
\hline 100 to 199 acres & 158,005 & $11.6 \%$ & $|4|, 875$ & $10.7 \%$ \\
\hline 200 to 499 acres & 163,027 & $12.0 \%$ & 143,776 & $10.8 \%$ \\
\hline 500 to 999 acres & 90,412 & $6.6 \%$ & 82,211 & $6.2 \%$ \\
\hline I,000 to 1,999 acres & 54,281 & $4.0 \%$ & 53,728 & $4.0 \%$ \\
\hline 2,000 acres or more & $2|, 4| \mid$ & $1.6 \%$ & 27,093 & $2.0 \%$ \\
\hline
\end{tabular}

Source: The 2007 Census of Agriculture, National Agricultural Statistics Service, US Department of Agriculture, Volume I, Table 8. 


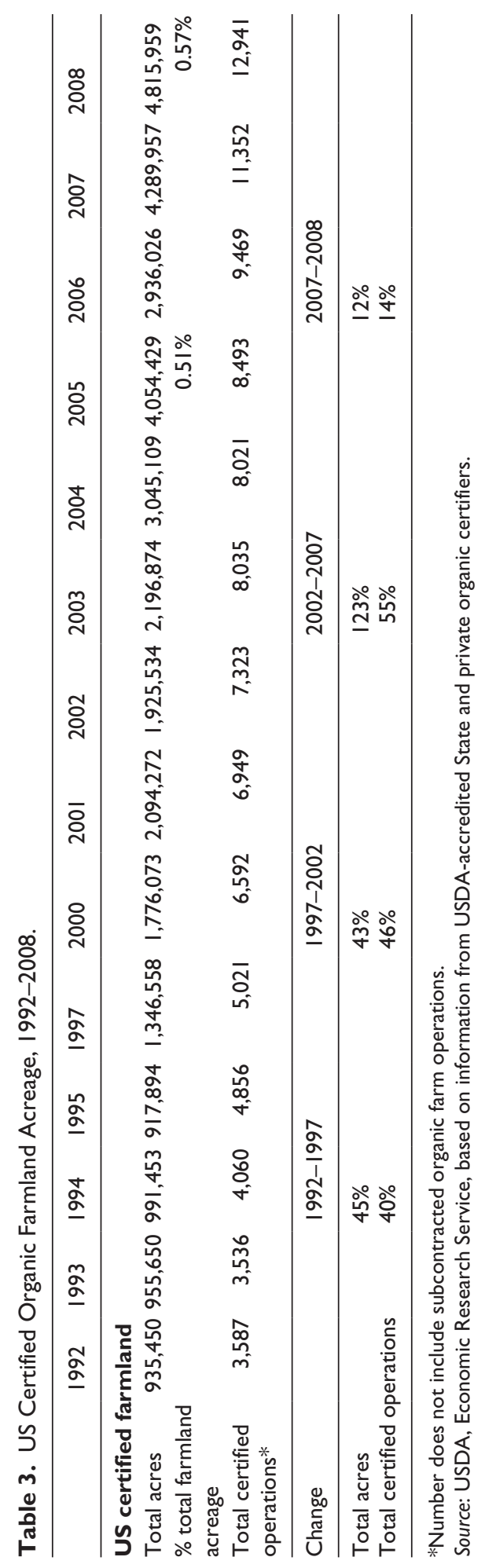


Table 4. USDA-Accredited Organic Certification Programs Active in 2002 through 2008!.

\begin{tabular}{|c|c|c|c|c|c|c|c|c|c|c|}
\hline \multirow[t]{2}{*}{ Certifier } & \multirow[t]{2}{*}{ Headquarters } & \multirow{2}{*}{$\begin{array}{l}\text { USDA } \\
\text { Accreditation } \\
\text { Date }\end{array}$} & \multicolumn{8}{|c|}{ Producers certified } \\
\hline & & & 2002 & 2003 & 2004 & 2005 & 2006 & 2007 & 2008 & \\
\hline \multicolumn{11}{|l|}{ State } \\
\hline $\begin{array}{l}\text { County of Monterey } \\
\text { Agricultural } \\
\text { Commissioner's Office }\end{array}$ & Salinas, CA & $4 / 29 / 2002$ & 12 & 10 & 12 & 12 & 8 & 11 & 8 & \\
\hline $\begin{array}{l}\text { Marin County } \\
\text { Department of } \\
\text { Agriculture }\end{array}$ & Novato, CA & $4 / 29 / 2002$ & - & 37 & 36 & 36 & 55 & 57 & & 60 \\
\hline $\begin{array}{l}\text { Idaho Dept. of } \\
\text { Agriculture }\end{array}$ & Boise, ID & $4 / 29 / 2002$ & 153 & 149 & 170 & 162 & 162 & 188 & & 203 \\
\hline $\begin{array}{l}\text { lowa Dept. of Agriculture } \\
\text { and Land Stewardship }\end{array}$ & $\begin{array}{l}\text { Des Moines, } \\
\text { IA }\end{array}$ & $4 / 29 / 2002$ & 116 & 136 & $|5|$ & 174 & 208 & 211 & & 300 \\
\hline $\begin{array}{l}\text { Maryland Dept. of } \\
\text { Agriculture }\end{array}$ & Annapolis, MD & $4 / 29 / 2002$ & 75 & 75 & 59 & 60 & 93 & 89 & & 111 \\
\hline $\begin{array}{l}\text { Montana Dept. of } \\
\text { Agriculture }\end{array}$ & Helena, MT & $4 / 29 / 2002$ & - & 75 & 67 & 86 & 91 & 98 & & 98 \\
\hline $\begin{array}{l}\text { New Hampshire Dept. of } \\
\text { Agriculture }\end{array}$ & Concord, NH & $4 / 29 / 2002$ & 59 & 70 & 76 & 92 & 92 & 119 & & 102 \\
\hline $\begin{array}{l}\text { Texas Dept. of } \\
\text { Agriculture }\end{array}$ & Austin, TX & $4 / 29 / 2002$ & 129 & 129 & 147 & 159 & 203 & 169 & & 203 \\
\hline $\begin{array}{l}\text { Utah Department of } \\
\text { Agriculture }\end{array}$ & $\begin{array}{l}\text { Salt Lake City, } \\
\text { UT }\end{array}$ & $4 / 29 / 2002$ & - & - & - & - & - & 36 & & 36 \\
\hline $\begin{array}{l}\text { Virginia Dept. of } \\
\text { Agriculture \& Consumer } \\
\text { Services }{ }^{2}\end{array}$ & Richmond, VA & $4 / 29 / 2002$ & 120 & - & - & - & - & - & - & \\
\hline $\begin{array}{l}\text { Washington State } \\
\text { Department of } \\
\text { Agriculture }\end{array}$ & Olympia, WA & $4 / 29 / 2002$ & 544 & 553 & 558 & 552 & 554 & 629 & & 697 \\
\hline $\begin{array}{l}\text { Nevada Dept. of } \\
\text { Agriculture }\end{array}$ & Reno, NV & $5 / 13 / 2002$ & 18 & 22 & 21 & 21 & 30 & 34 & & 36 \\
\hline $\begin{array}{l}\text { New Mexico Organic } \\
\text { Commodity Commission }\end{array}$ & $\begin{array}{l}\text { Albuquerque, } \\
\text { NM }\end{array}$ & $5 / 29 / 2002$ & 93 & 93 & 78 & 99 & 152 & 184 & & 193 \\
\hline $\begin{array}{l}\text { Colorado Dept. of } \\
\text { Agriculture }\end{array}$ & $\begin{array}{l}\text { Lakewood, } \\
\text { CO }\end{array}$ & $10 / 16 / 2002$ & 186 & 80 & 92 & 99 & 112 & 125 & & 138 \\
\hline $\begin{array}{l}\text { Rhode Island Dept. } \\
\text { of Environmental } \\
\text { Management }\end{array}$ & Providence, RI & $10 / 22 / 2002$ & 9 & 9 & 10 & 18 & 17 & 21 & & 22 \\
\hline $\begin{array}{l}\text { Oklahoma Dept. of } \\
\text { Agriculture }\end{array}$ & $\begin{array}{l}\text { Oklahoma } \\
\text { City, OK }\end{array}$ & $12 / 6 / 2002$ & 15 & 21 & 30 & 28 & 34 & 56 & & 56 \\
\hline $\begin{array}{l}\text { Mississippi Dept. } \\
\text { of Agriculture and } \\
\text { Commerce }\end{array}$ & Meridian, MS & $6 / 1 / 2004$ & - & - & - & 6 & 23 & 22 & & 22 \\
\hline $\begin{array}{l}\text { Louisiana Dept. of } \\
\text { Agriculture }\end{array}$ & $\begin{array}{l}\text { Baton Rouge, } \\
\text { LA }\end{array}$ & $9 / 23 / 2004$ & - & - & - & 10 & 12 & 12 & & 12 \\
\hline $\begin{array}{l}\text { Kentucky Department of } \\
\text { Agriculture }\end{array}$ & Frankfort, KY & $1 / 13 / 2006$ & - & - & - & - & - & - & & 55 \\
\hline
\end{tabular}


Table 4. (Continued)

\begin{tabular}{|c|c|c|c|c|c|c|c|c|c|}
\hline \multirow[t]{2}{*}{ Certifier } & \multirow[t]{2}{*}{ Headquarters } & \multirow{2}{*}{$\begin{array}{l}\text { USDA } \\
\text { Accreditation } \\
\text { Date }\end{array}$} & \multicolumn{7}{|c|}{ Producers certified } \\
\hline & & & 2002 & 2003 & 2004 & 2005 & 2006 & 2007 & 2008 \\
\hline $\begin{array}{l}\text { Yolo County Department } \\
\text { of Agriculture }\end{array}$ & Woodland, CA & $1 / 22 / 2006$ & - & - & - & - & 4 & 4 & 11 \\
\hline \multicolumn{10}{|l|}{ Private } \\
\hline $\begin{array}{l}\text { California Certified } \\
\text { Organic Farmers }\end{array}$ & $\begin{array}{l}\text { Santa Cruz, } \\
\text { CA }\end{array}$ & $4 / 29 / 2002$ & $\mathrm{I}, 040$ & $\mathrm{I}, \mathrm{I} 47$ & 994 & 1,062 & $\mathrm{I}, 523$ & $\mathrm{I}, 808$ & 2,007 \\
\hline $\begin{array}{l}\text { California Organic } \\
\text { Farmers Association }\end{array}$ & $\begin{array}{l}\text { North Modoc, } \\
\text { CA }\end{array}$ & $4 / 29 / 2002$ & II & 11 & 22 & 22 & 42 & 43 & 48 \\
\hline $\begin{array}{l}\text { Clemson Univ. Fertilizer } \\
\text { and Seed Certification } \\
\text { Services }\end{array}$ & Pendleton, SC & $4 / 29 / 2002$ & - & 4 & 6 & 8 & 14 & 64 & 25 \\
\hline $\begin{array}{l}\text { Demeter Association's } \\
\text { Stellar Certification } \\
\text { Services }\end{array}$ & Aurora, NY & $4 / 29 / 2002$ & 57 & 75 & 75 & 75 & 73 & 76 & 76 \\
\hline $\begin{array}{l}\text { FOG's Quality } \\
\text { Certification Services }\end{array}$ & Gainesville, FL & $4 / 29 / 2002$ & 178 & 221 & 140 & 140 & 140 & 300 & 300 \\
\hline $\begin{array}{l}\text { Georgia Crop } \\
\text { Improvement Association }\end{array}$ & Athens, GA & $4 / 29 / 2002$ & I & 8 & 23 & 35 & 40 & 79 & 41 \\
\hline Global Organic Alliance & $\begin{array}{l}\text { Bellefontaine, } \\
\mathrm{OH}\end{array}$ & $4 / 29 / 2002$ & 146 & 262 & 306 & 284 & 341 & 415 & 412 \\
\hline $\begin{array}{l}\text { Guaranteed Organic } \\
\text { Certification Agency }\end{array}$ & Fallbrook, CA & $4 / 29 / 2002$ & - & 251 & 256 & 333 & 326 & 408 & 384 \\
\hline $\begin{array}{l}\text { Hawaii Organic Farmers } \\
\text { Association }\end{array}$ & Haiku, HI & $4 / 29 / 2002$ & 110 & 110 & 108 & 98 & 128 & 78 & 115 \\
\hline $\begin{array}{l}\text { Integrity Certified } \\
\text { International, Inc. }\end{array}$ & Bellevue, NE & $4 / 29 / 2002$ & 12 & 12 & 28 & 35 & - & - & - \\
\hline Indiana Certified Organic & Clayton, IN & $4 / 29 / 2002$ & 154 & 182 & 201 & 202 & 265 & 316 & 269 \\
\hline $\begin{array}{l}\text { International Certification } \\
\text { Services-Farm Verified } \\
\text { Organic }\end{array}$ & Medina, ND & $4 / 29 / 2002$ & 269 & 258 & 249 & 231 & 233 & 277 & 294 \\
\hline $\begin{array}{l}\text { Maharishi Vedic Organic } \\
\text { Agriculture Institute }\end{array}$ & Fairfield, IA & $4 / 29 / 2002$ & - & 4 & 3 & 2 & - & - & - \\
\hline $\begin{array}{l}\text { Minnesota Crop } \\
\text { Improvement Association }\end{array}$ & St. Paul, MN & $4 / 29 / 2002$ & 3 & 14 & 20 & 22 & 28 & 28 & 29 \\
\hline $\begin{array}{l}\text { Midw est Organic } \\
\text { Services Association }\end{array}$ & Viroqua, WI & $4 / 29 / 2002$ & 490 & 621 & 547 & 546 & 672 & 862 & 1026 \\
\hline $\begin{array}{l}\text { Northeast Organic } \\
\text { Farmers Assn.-MA }\end{array}$ & $\begin{array}{l}\text { West Hatfield, } \\
\text { MA }\end{array}$ & $4 / 29 / 2002$ & 90 & 90 & 120 & 119 & 118 & 133 & 138 \\
\hline $\begin{array}{l}\text { Northeast Organic } \\
\text { Farmers Assn.-NJ }\end{array}$ & Pennington, NJ & $4 / 29 / 2002$ & 68 & 55 & 56 & 54 & 54 & 60 & 64 \\
\hline $\begin{array}{l}\text { Northeast Organic } \\
\text { Farmers Assn.-NY }\end{array}$ & $\begin{array}{l}\text { Binghamton, } \\
\text { NY }\end{array}$ & $4 / 29 / 2002$ & 195 & 287 & 268 & 360 & 422 & 543 & 583 \\
\hline $\begin{array}{l}\text { Nutriclean (formerly } \\
\text { Scientific Certification } \\
\text { Systems) }\end{array}$ & Oakland, CA & $4 / 29 / 2002$ & 5 & 5 & - & - & - & - & - \\
\hline $\begin{array}{l}\text { Ohio Ecological Food \& } \\
\text { Farming Association }\end{array}$ & $\begin{array}{l}\text { West Salem, } \\
\mathrm{OH}\end{array}$ & $4 / 29 / 2002$ & 195 & 214 & 179 & 250 & 250 & 404 & 661 \\
\hline
\end{tabular}


Table 4. (Continued)

\begin{tabular}{|c|c|c|c|c|c|c|c|c|c|}
\hline \multirow[t]{2}{*}{ Certifier } & \multirow[t]{2}{*}{ Headquarters } & \multirow{2}{*}{$\begin{array}{l}\text { USDA } \\
\text { Accreditation } \\
\text { Date }\end{array}$} & \multicolumn{7}{|c|}{ Producers certified } \\
\hline & & & 2002 & 2003 & 2004 & 2005 & 2006 & 2007 & 2008 \\
\hline Oregon Tilth & Salem, OR & $4 / 29 / 2002$ & 379 & 381 & 391 & 398 & 357 & 578 & 603 \\
\hline Organic Certifiers & Ventura, CA & $4 / 29 / 2002$ & 50 & 79 & 72 & 75 & 106 & 123 & 160 \\
\hline $\begin{array}{l}\text { Organic Crop } \\
\text { Improvement Association }\end{array}$ & Lincoln, NE & $4 / 29 / 2002$ & 1,136 & 953 & $\mathrm{I}, 048$ & 985 & 872 & 852 & 833 \\
\hline $\begin{array}{l}\text { Organic Forum } \\
\text { International, Inc. }\end{array}$ & $\begin{array}{l}\text { Paynesville, } \\
\text { MN }\end{array}$ & $4 / 29 / 2002$ & 21 & 21 & - & - & - & - & - \\
\hline $\begin{array}{l}\text { Pennsylvania Certified } \\
\text { Organic }\end{array}$ & $\begin{array}{l}\text { Centre Hall, } \\
\text { PA }\end{array}$ & $4 / 29 / 2002$ & 202 & 223 & 270 & 287 & 287 & 320 & 408 \\
\hline $\begin{array}{l}\text { Quality Assurance } \\
\text { International }\end{array}$ & San Diego, CA & $4 / 29 / 2002$ & 408 & 408 & 179 & 256 & 379 & 344 & 346 \\
\hline $\begin{array}{l}\text { Organic Growers of } \\
\text { Michigan }^{6}\end{array}$ & $\begin{array}{l}\text { Grand Rapids, } \\
\text { MI }\end{array}$ & $5 / 13 / 2002$ & 39 & 55 & - & - & - & - & - \\
\hline Pro-Cert ${ }^{7}$ & $\begin{array}{l}\text { Saskatchewan, } \\
\text { Canada }\end{array}$ & $5 / 24 / 2002$ & - & - & - & - & - & - & 82 \\
\hline $\begin{array}{l}\text { Maine Organic Farmers \& } \\
\text { Gardeners Association }\end{array}$ & Unity, ME & $6 / 3 / 2002$ & 259 & 259 & 286 & 286 & 316 & 349 & 632 \\
\hline $\begin{array}{l}\text { North Carolina Crop } \\
\text { Improvement Association }\end{array}$ & Raleigh, NC & $7 / 9 / 2002$ & 12 & 21 & 25 & 33 & 38 & 35 & 68 \\
\hline $\begin{array}{l}\text { California Crop } \\
\text { Improvement Association }\end{array}$ & Davis, CA & $8 / 7 / 2002$ & - & - & 12 & 12 & - & - & - \\
\hline $\begin{array}{l}\text { Northeast Organic Farmers } \\
\text { Vermont Organic Farm Rich }\end{array}$ & $\begin{array}{l}\text { s Assn.-VT/ } \\
\text { hmond, VT }\end{array}$ & $9 / 24 / 2002$ & 253 & 289 & 332 & 366 & 394 & 487 & 535 \\
\hline Natural Food Certifiers & Scarsdale, NY & $10 / 8 / 2002$ & - & 7 & I & I & - & - & - \\
\hline Certified Organic, Inc. & Keosauqua, IA & II/I2/2002 & - & - & 7 & 7 & 20 & 24 & 24 \\
\hline $\begin{array}{l}\text { American Food Safety } \\
\text { Institute }^{3}\end{array}$ & Davis, CA & $2 / 10 / 2003$ & - & 3 & - & - & - & - & - \\
\hline Global Culture & $\begin{array}{l}\text { Crescent City, } \\
\text { CA }\end{array}$ & $4 / 14 / 2003$ & - & 26 & 56 & 76 & 67 & 71 & 82 \\
\hline OneCert & Lincoln, NE & $4 / 22 / 2003$ & - & - & 21 & 89 & 114 & 143 & 207 \\
\hline Primuslabs.com & $\begin{array}{l}\text { Santa Maria, } \\
\text { CA }\end{array}$ & $1 / 22 / 2006$ & - & - & - & - & - & 2 & I \\
\hline $\begin{array}{l}\text { Agricultural Services } \\
\text { Certified Organic }\end{array}$ & Salinas, CA & $4 / 7 / 2006$ & - & - & - & - & - & 22 & 22 \\
\hline $\begin{array}{l}\text { Nature's International } \\
\text { Services }\end{array}$ & Viroqua, WI & $2 / 12 / 2007$ & - & - & - & - & - & 43 & 103 \\
\hline $\begin{array}{l}\text { US total, state and private } \\
\text { groups }\end{array}$ & & & 7,312 & 8,015 & 7,808 & 8,363 & 9,469 & II,352 & $|2,94|$ \\
\hline
\end{tabular}

'USDA's Economic Research Service only tracks operations certified by US-based certifiers. Numbers may not reflect subcontracted operations. A current list of certifiers is maintained by USDA's National Organic Program, www.ams. usda.gov/nop.

${ }^{2}$ Dropped certification program in 2003.

${ }^{3}$ Accreditation revoked in 2006, no information available for 2004 or 2005.

${ }^{4}$ Certifier did not provide certified acreage and livestock estimates.

${ }^{5}$ Dropped certification program in 2005, no information available for 2004 or 2005.

6Dropped certification program in 2006, no information available for 2004 or 2005.

${ }^{7}$ Canadian certifier that provides services in the US.

Source: USDA, Economic Research Service, based on information from USDA-accredited State and private organic certifiers. 


\section{Resource Partitioning Theory and Identity Movements}

The case of organic farming is interesting from a theoretical perspective because it does not conform to the pattern of identity movement-led resource partitioning noted in previous studies. As the theory holds, identity movements nourished by post-materialist sentiments (Ingelhart, 1990) seek to challenge dominant logics and conformity pressures and inspire cultural change (Buechler, 1995; Mezias \& Mezias, 2000; Pichardo, 1997; Weber, Heinze, \& DeSoucey, 2008). They trigger partitioning by increasing the dimensionality of the resource space by introducing new tastes, needs or identities to it. They typically seize industries related to culture (Mezias \& Mezias, 2000), food (e.g., Carroll \& Swaminathan, 2000) or media (e.g., Greve et al., 2006; Pozner \& Rao, 2006), and emerge as a reaction to the homogenization created by market concentration in the hands of a few dominant generalists. Such reactions translate into what Carroll and Swaminathan (2000) term "anti-mass-production cultural sentiment," a mindset embodying opposition to mass society, its production techniques, and its corporate organization (Peterson, 1997). This sentiment of anti-mass-production is typically embodied in the definition of the specialists' organizational form identity, which by its nature becomes oppositional to that of the generalists.

An organizational form identity is a recognizable pattern or a set of signals that takes on rulelike standing and is governed by an externally maintained normative code (Polos, Hannan, \& Carroll, 2002). That is, it depends on the perceptions, opinions and actions of social actors as they encounter the organization. Organizational forms and codes that are perceived as meaningfully and sharply defined eventually become institutionalized, which enhances their viability by bestowing privileged status, access to resources, and thereby the ability to fend off challenges (Hannan, 1991; Polos et al., 2002). Because of the organizational form's rule-like status, visible violations are normatively sanctioned by identity movement adherents - typically, highly identified and dedicated social actors who are familiar with the definitions of the organizational form and who are ready to sanction, boycott or ridicule organizations who violate normative codes or attempt to masquerade as specialists (Carroll \& Swaminathan, 2000).

Identity movements are, therefore, an important driver of resource partitioning, not only because they increase the dimensionality of the resource space, but also because they engender the personal identification that leads participants to sanction violations of the organizational form identity (Carroll \& Swaminathan, 2000). This mechanism - the imposition of sanctions for violation of organizational form identity - is the key to understanding the difference between the organic farming case, where resource partitioning has not been stable, and previous cases, where markets are partitioned in equilibrium. That is, given that organizational forms vary in sharpness or specificity (McKendrick \& Carroll, 2001), only when specialist organizations develop a sharply defined specialist form identity are they able to forestall competition from generalists, who by definition violate the normative code associated with the specialist form and are therefore not capable of meaningfully competing in the specialist space. Greve and colleagues confirm this with regard to micro-radio: "LPFM stations were the result of a broad identity movement, but were a tightly defined organizational form that had to conform to a regulatory code of conduct" (Greve et al., 2006, p. 808). Failure to generate a sharply defined specialist form identity creates the risk that violations will not be recognized and that generalists masquerading as specialists will not be punished. More specifically, we note that the ability of identity movements to narrowly define organizational forms and ensure market partitioning depends on a number of mechanisms which we find are critical in the partitioning of markets. In both the micro-radio and the micro-brew cases, identity movements spurred the establishment of a narrow and sharply defined specialist form identity, and in both cases, the mechanisms underlying the identity movements were similar. 


\section{Mechanisms Underlying Partitioning}

We now turn to the mechanisms underlying market partitioning before examining the extent to which they differ in the organic farming movement as compared to the micro-brew and microradio movements. They include: the formulation of common goals pursued by movement adherents; the use of oppositional framing and discourse to portray generalists and specialists; the role of mobilization in obtaining a heterogeneous group of movement adherents; and the establishment and nurturing of a social and community infrastructure supportive of the emergence of specialist organizations.

Formulating common goals. Identity movements can only induce market partitioning when they articulate a clearly formulated, consistent and commonly shared movement goal. Studies of the micro-radio movement show how the mobilization of micro-radio activists was based on a common goal (Greve et al., 2006; Pozner \& Rao, 2006), specifically to "challenge the domination of radio by corporate chains by putting new voices onto the airwaves, to attract an audience for these new voices, and ultimately to reduce the influence of chain-owned radio stations" (Greve et al., 2006, p. 809). Similarly, in their widely cited study on the proliferation of US micro-breweries, Carroll and Swaminathan (2000) documented how movement adherents united to distance themselves from corporate breweries and beer. By recognizing and formulating common goals, movement adherents unite and develop a "we-feeling," a sense of community that encourages mobilization, collective action, and loyalty in the pursuit of the common cause.

Oppositional framing and discourse. The case of micro-radio also highlights the importance of identity movements' use of framing and discourse that fosters expectations and inspires action, thereby delineating and reifying the boundaries between generalists and specialists. In other words, identity movements "depend on a salient enemy of concentrated mass producers, public discourse raising this concentration as a social problem requiring entrepreneurial problem solving, and community resources for creating anti-mass production organizations" (Greve et al., 2006, p. 809). Microradio activists were adept at mobilizing movement participants by framing the generalist form identity as a common enemy against which everybody who cared about the cause must rally (Greve et al., 2006; Pozner \& Rao, 2006). They also used carefully crafted discourse to portray the specialist form identity as a solution to the homogenization problem emanating from increasing market concentration and generalist domination.

A common enemy is important to market partitioning because it drives the anti-mass-production sentiments that lead consumers to buy specialists' products. Anti-mass-production sentiment consists of two distinct yet related dimensions (Carroll \& Swaminathan, 2000; Greve et al., 2006). On the one hand, it is directed at opposing mass producers, or the dominant generalists and their products. For example, micro-brew consumers opposed major corporate breweries and what they characterized as "industrial beer." Similarly, in the micro-radio case, the opposition was directed at chain-owned radio stations and the increasingly mainstream content they aired.

On the other hand, anti-mass-production cultural sentiment is also directed against mass production: the creation of homogeneous, undifferentiated products on a large scale. Opposition to mass-produced goods is spurred by consumers' need for products that convey a sense of exclusivity or represent an alternative to mainstream tastes, and is fueled by identity enhancement and identity expression motives. In the micro-brew case, Carroll and Swaminathan (2000) note that consumption of specialty beer is driven by consumers' need for products that convey a "sense of expertise, sophistication and refinement." In the micro-radio case, activists are driven by a need for 
increasing diversity by putting new voices onto the airwaves (Greve et al., 2006). In both cases the common denominator seems to revolve around the notion of being different from others.

Both dimensions of anti-mass-production sentiment are tied to the means of production. Specialists proliferate because they respond to identity movement adherents' demands for authentic products delivered by producers embodying a specific, oppositional organizational form identity, which involves attributes such as unconventionality as well as purity, local craftsmanship, and traditional manufacturing. Because generalists cannot offer such products, the method of production associated with the specialist form identity is the primary driver of partitioning: movement adherents delineate boundaries by publicly criticizing generalists' production methods. For example, micro-radio activists criticized chain-owned broadcasters for replicating formats nationwide, for replacing local radio personalities with syndicated programming, and for eliminating local news departments (Greve et al., 2006). Public critique of generalists and their production methods helps reify the boundaries between the generalist and specialist form identities.

Not surprisingly, therefore, part of specialists' appeal hinges upon small-scale production, and both micro-brews and micro-radio stations have limits to growth imprinted in their organizational form identities. In the micro-brew case, small-scale production is ensured by limits to production capacity; the American Brewers Association defines a "micro-brewery" as a brewery whose production capacity does not exceed 15,000 barrels (Brewers Association, 2011). In the micro-radio case, the limitations concern the broadcast radius of a maximum of 3.5 miles and are achieved by restricting the technical specifications of micro-radio stations (Greve et al., 2006). As Carroll and Swaminathan (2000) note, small-scale production is associated with higher product quality in the eyes of consumers, who have great faith in the ability of small organizations to produce and deliver high-quality specialty products. Such attributions are socially constructed, however; as demonstrated in the micro-brew case, quality attributions are more about specialist identity and method of production than objective product quality. Carroll and Swaminathan (2000) document this with reference to perfectly content beer drinkers who look "crestfallen" when told that their specialty brews are actually produced by mass brewers. This suggests that those involved in identity movements make their buying decisions based on organizational form identity, rather than on product characteristics alone.

Mobilizing a broad base of adherents. Given the requirement of some unifying common enemy or goal, it is important to consider how much heterogeneity movements can tolerate while remaining a viable trigger for market partitioning. As studies of other movements suggest, increases in both the number and heterogeneity of participants and organizations help sustain movements (McAdam, 1999; Oberschall, 1973). Heterogeneity fuels movements' success by creating appeal to different constituencies, mobilizing different strata of the population, and increasing the number of alternatives for potential members and benefactors (McAdam, 1999; Oberschall, 1973). In addition, movement growth attributable to spillover from other movements seems to help movements succeed (Meyer \& Whittier, 1994), provided that the goals pursued by the different movement participants are nested, if not common. For example, in the micro-radio case, the movement's narrow goal was to provide voices on the air in order to serve previously underserved segments. This goal was nested in the broader, more general goal of reducing the dominance of chains in the radio market (Greve et al., 2006). While the two goals appear to have a different effect on the distribution of resources between generalists and specialists, their differences did not impede the success of micro-radio, but benefited the movement by reinforcing boundaries between generalists and specialists. Thus a broad base of heterogeneous movement adherents and organizations pursuing similar goals benefit the specialist form identity and sustain partitioning. 
Nurturing a supportive social and community infrastructure. A final mechanism decisive for how identity movements impact market partitioning concerns the nurturing of a supportive and dense social and community infrastructure. Greve and colleagues (2006) emphasize the role of community infrastructure and trained organization builders in spurring specialist proliferation. They claim that institutional actors such as voluntary associations, work groups, and other organizations serve as building blocks of identity movements. They help adapt goals to serve the needs of emergent causes and strategically frame issues in a way that bridges their connection to formal institutions. Formal institutions, such as government agencies, regulators, and certification bodies, therefore help institutionalize the oppositional specialist form identities and sustain market partitioning. In particular, certification organizations have been shown to encourage the development of new market sectors by legitimating new organizational form identities, particularly through differentiation from existing alternatives (Bartley, 2003; Lee, Sine, \& Tolbert, 2011; Lounsbury, Ventresca, \& Hirsch, 2003; Rao, 1994).

Similarly, dense and redundant social ties among movement adherents aid partitioning by allowing for rapid and pervasive information flow within the social network. Such efficient exchange of information not only aids specialists in promoting their organizational form identity while educating and challenging consumer perceptions, but is also indispensable for unmasking inauthentic generalists disguised as specialists. Carroll and Swaminathan (2000) find that identity management by generalists is difficult when movement adherents are strongly connected and form a dense network of self-styled experts. Because information is exchanged rapidly among micro-brew aficionados, the status of craft-style beers produced by mass brewers plummets along with the credibility of the generalist brewer. Hence, a thick and interconnected network of movement followers sustains market partitioning by exposing and sanctioning code violations and enhancing specialists' form identity (Carroll \& Swaminathan, 2000).

\section{Unpacking the Organic Case}

In our search to better understand how identity movements impact market partitioning, we reason that, because the mechanisms underlying movements vary, identity movements may differ in their ability to generate a sharply defined specialist form identity, induce sanctioning of its visible form and, hence, engender stable market partitioning. We thus identify the key stages of the organic movement and examine the mechanisms related to goals, framing, mobilization, and nurturing of social infrastructure as the organic movement unfolds. We are particularly interested in how the organic case differs from the micro-brewery and micro-radio cases, as well as how these differences change over time, as a way to understand the organic movement's ability to create and sustain a partitioned market.

\section{Stage I: Towards a Partitioned Market}

In the case of organic farming, the degree and stability of market partitioning seems to have changed along with changes in the underlying organic farming movement. In the first stage, which started with the rise of organic farms inspired by early activists' ideals and which lasted through the 1980s farm crisis, the mechanisms driving the organic movement were very similar to those found in the micro-radio and the micro-brew cases. As conventional farm productivity increased, fields grew bigger, farms larger, agribusiness consolidated, and - just as resource partitioning theory predicts - small, specialist organic farms sprouted up in response. 
In its initial stages, the movement professed the clearly defined goal of combating the increased commoditization and industrialization of agriculture. Early movement adherents may have differed in their precise definition of organic methods, but they were unequivocal in their opposition to industrial methods, which they thought unnatural and damaging to people, animals and the Earth. Their production methods were sharply opposed to those of conventional generalists, employing craft production versus mechanization, traditional varieties versus hybrid and engineered seeds, natural versus synthetic fertilizers and pesticides. These methods were reflective of the ideology of each category of producer: staying close to the Earth and preserving its integrity in the case of movement adherents, increasing scale and profitability while satisfying growing food demands for generalist agribusiness. Small organic farmers and the holistic techniques they supported were positioned as the solution to the homogenization and health problems created by conventional agriculture. Consequently, the common enemy was readily identified as large conventional farmers who were interested in growing both production scale and profit at the expense of the environment and consumer wellbeing.

As the organic movement gained a broader base of adherents from the environmental movement in the 1960s and 1970s, the rhetoric and unity of the organic movement seemed to remain. Even if environmental groups added to movement heterogeneity, they pursued similar goals, as the discourse on threats to individual and environmental health were closely related. Thus, in the first stage of the organic farming movement, organic farmers fairly successfully developed oppositional strategies and united against conventional farmers in the pursuit of the common goal of promoting more balanced, chemical-free, healthy farming methods. This assertion is reinforced by consumers' reactions to organic products in this first stage of the movement. Long before organics and environmentalism became trendy, the social infrastructure of the organic movement was supportive of the development of an organic form identity. Contemporary and historical accounts indicate that early organic consumers were a zealous community of followers who did not shy away from paying higher prices for products they considered more natural, healthy, and ethical. Together with organic farmers, they formed a tight-knit community of devotees who identified strongly with the movements' ideals. Displays of symbolic consecration and loyalty are probably best illustrated by food festivals and farmers' markets. In the first stage of the organic movement, therefore, the market for farming produce can be described as partitioned; specialists served well-defined niches which generalists were not interested to exploit, hence specialists and generalists coexisted without competing directly.

However, although organic movement adherents successfully opposed large conventional farmers and their industrial production techniques, they were less successful at precisely defining their own production methods and, consequently, a clear organizational form identity. For example, Rudolph Steiner, an early advocate of organic farming, advocated "biodynamic agriculture" and emphasized the farmer's role in guiding and balancing the interaction of the animals, plants and soil, whereas Jerome Rodale's definition of organic revolved around the idea of a symbiotic farm system and the return to sustainable agriculture (see Table 1). While these meanings of organic production certainly overlap, they do not converge towards a single definition. More importantly, they do not include a precise definition of which techniques, production processes, and inputs constitute organic farming and which do not. It is this shortcoming that may have encouraged a mushrooming of organic farms and fuzzy definitions of organic production, which ultimately compromised the stability of market partitioning.

Moreover, although movement adherents opposed mass production techniques, their opposition to large-scale farming was not explicitly articulated, but rather implicit in their depiction of organic 
farming methods. Consequently, the idea that organic farms were by definition small-scale operations was not a constitutive element of the organic organizational form identity. Limits to growth are salient not only because they make the specialist identity more readily recognizable and violations more visible, but also because the consumption of goods made by small-scale producers induces the sense of exclusivity and distinctness pertinent to identity expression and identity enhancement motives. Had the organic ideology explicitly dismissed the possibility of bringing organic farming to scale, expansionists would have had no traction in framing themselves as authentic organic farmers. Moreover, organic consumers would have seen scalability as a violation of the organic form identity, further discouraging expansionists from entering the specialist resource space. Without such technical limits to growth, however, the already blurry definition of organic production in the early stages of the movement prevented the specialist form identity from becoming fully institutionalized, leading to problems as the movement entered its second stage. In sum, the weak definition of the organic organizational form prevented the movement from articulating clear, common goals and framing their movement in strong opposition to large-scale, conventional farming, and thus compromised the stability of market partitioning.

\section{Stage 2: Eroding Boundaries and De-Partitioning}

The failure of the early organic movement to generate a clearly oppositional and sharply defined specialist form identity ultimately led to market de-partitioning, allowing conventional farmers to approach organic farmers' resource space in the late 1980s and early 1990s and erode the boundaries between generalists and specialists. In this second stage of market development, the movement became increasingly heterogeneous and developed extremely loose social networks, leading to conflicting goals and rhetorical framing on the part of different movement fractions. Subsequent appeals to existing institutions did not reinforce market partitioning, but rather undermined the specialist identity form by encouraging competition between what would have previously been labeled generalists and specialists.

The process of de-partitioning began with the bifurcation of the organic movement in the late 1980s and 1990s into the agrarian and the expansionist camps. The introduction of heterogeneous actors with quite divergent goals compromised two important levers of identity-movement-driven market partitioning. First, it compromised the pursuit of the common goals thereby weakening the movement's potential to sustain the sense of community necessary for participants to coalesce. This impaired organic farmers' ability to frame discourse in terms of oppositional strategies to conventional farmers, whose methods became less and less distinguishable from those of expansionists. As the image of conventional farming as the common enemy faded and the scale of organic farming grew, agrarians found it increasingly difficult to articulate the organic form identity and its method of production as a solution to generalist dominance. In fact, generalist dominance became less of a problem for the modal organic consumer; even though produce could be labeled organic without strict adherence to movement ideals, at least some of those ideals are upheld in any certified organic production. The availability of organic fruits and vegetables raised in monoculture and sold in national supermarket chains benefits a tremendous number of casual consumers as well as the Earth, in terms of reduced use of synthetic fertilizers and pesticides. Once the problem agrarians fought could no longer be shown in such sharp relief, organic as a specialist form identity lost some of its raison d'être, compromising both common goals and oppositional framing.

As it turned out, malleability of the specialist form identity enabled expansionists to reach out to formal institutions responsible for defining and enforcing regulatory standards more successfully than the agrarians. As in the micro-brew and micro-radio cases, expansionists and agrarians 
relied on existing community infrastructures in their struggle to define the term organic. Agrarians pushed for standards at both the local (e.g., California Certified Organic Farmers certification) and national levels (e.g., the farm bill's inclusion of the Organic Foods Production Act). Lack of unanimity with respect to the organic form identity, however, resulted in competing regimes within the agrarian camp, which were ultimately unable to protect organic farmers from competition. Moreover, lack of unanimity with respect to what organic production means may have helped the expansionist camp to win the battle thanks to its stronger resource pool, connections, and lobbying strategies. Thus the poorly nurtured social infrastructure encouraged market de-partitioning, in sharp contrast to the micro-radio case.

Even more important was the perception of what organics meant to consumers. By failing to agree upon a clear definition of organic farming, agrarians missed an opportunity to educate consumers about what constituted the organic form identity, thereby engendering oppositional strategies to organizations that did not fit the bill. As a result, code violations could not be readily recognized by any but the most educated and motivated consumers, and therefore went unsanctioned by the vast majority of organic consumers. The inability of consumers to identify potential violations of the organic specialist form identity was not only a function of agrarians' missed opportunity, but also of changes to the composition of the organic consumer profile. In the 1990s and early 2000s the organic consumer profile expanded to include those who gravitated towards organic produce more for lifestyle than for ideological reasons. Thus, the more popular the organic movement became, the more it attracted individuals less inclined to publicly wave the flag for the movement's ideals, but for whom association with the movement was a symbolic sometimes unconscious - act requiring little knowledge or expertise about producer and product authenticity. In effect, the success of the organic movement in finding a broad base of support actually compromised its integrity and organizational form identity. This is in sharp contrast to the micro-brew and micro-radio cases, which seem not to have grown to the point where movement identification would pose a problem to the loyalty of its adherents.

Much in line with works on the formation of collective identities across stages of social movements (Benford \& Snow, 2000; Jasper, 1997; Polletta \& Jasper, 2001), it seems reasonable to assume that the growth of the organic movement engendered lower levels of collective identification with its ideals, making detecting and sanctioning violations of the original organic form identity increasingly difficult. Consequently, the boundaries between organic and conventional farming became eroded, leading eventually to what can be described as a de-partitioning of the market. By the end of this stage of market development, large, expansionist farms converted part of their operations to organic certification, resulting in direct competition between the small, ideologically conservative agrarian specialists and larger, ideologically liberal expansionist organic farms. In sum, it was the successful mobilization of a broad base of support and reaching out to social infrastructure combined with a lack of success in articulating common goals and an oppositional framing that led to market de-partitioning as the organic movement grew.

\section{Stage 3: Beyond Organic and Towards Re-Partitioning?}

Although the fruits of its labor are yet to be seen, a recent counter-movement has arisen at the edges of the organic movement which is poised to engender market re-partitioning. Under the umbrella of micro eco-farming (Adams, 2004), proponents of this new form of agriculture unite around the common goal of reviving the organic movement's initial ideals of sustainable, symbiotic agriculture and a back-to-the-land ethos. As one of the movement's proponents, endorsed by Rodale's New Farm Magazine (the outlet of the Rodale Institute), writes: "There is a change among those 
who farm in this century. They are not different than what they used to be. They are more of what they always were... They seem to have taken a long-lost power back" (Adams, 2004, p. 27). Micro eco-farmers are explicitly framing agribusiness as the problem to which their farming methods are a solution, effectively creating an oppositional discourse. "We don't need agribusiness to save us from starvation. Food is our excuse to co-create with nature instead of being passive recipients; to reach across species; to mingle with other humans; and to listen to an earthly problem" (Adams, 2004, p. 18).

In contrast to other organic farmers, micro eco-farmers are more consciously attempting to create an identity directly in opposition to large-scale farming, be it organic or conventional. They promote farming methods and use discourse that make of them even more "specialized specialists": instead of only local, they support homestead production; instead of simply natural, non-engineered seeds, they promote heirloom and open-pollinated fruit, grains, and vegetables; and instead of merely small-scale production, they emphasize self-sufficient farming (Center for the Micro Eco-Farming Movement, 2010). Moreover, rather than focusing on health, as did earlier organic proponents, micro eco-farmers invoke taste, shape, and flavor as motives for buying their produce which they claim is compromised in fruits and vegetables bred to survive lengthy shipping and storage (Center for the Micro Eco-Farming Movement, 2010).

By emphasizing both product characteristics and production methods, micro eco-farmers tap resources at the very edge of the resource space. As the term "micro eco-farming" suggests, the organizational form identity of this newly emerging specialist also echoes those of micro-brews and micro-radio, as it incorporates small-scale farming into its form definition. The movement defines itself as comprising "sustainable local mini-farms from urban greenhouses to backyard gardens to small rural 1 to 25-acre parcels" (Center for the Micro Eco-Farming Movement, 2010). This emerging counter-movement may thus generate a specialist identity sufficiently sharp and narrowly defined to become viable and sustain itself in the long run. By portraying themselves as a true and valid alternative to large-scale farming and by incorporating limits to organizational growth into its form identity, micro eco-farmers may satisfy the needs of those who are driven by both anti-mass producer and anti-mass production sentiments. This would enhance the new repartitioning of the farm produce market, with micro eco-farmers representing the true specialist form identity. Moreover, if micro eco-farmers are able to exploit their form identity successfully, they may become self-sustaining - as are micro-breweries - by reaping a price premium for their highly specialized products. They can do so by promoting their specialist form identity to signal uniqueness and refinement that neither organic nor conventional large-scale farmers are able to mimic. In addition, by portraying themselves as a true and viable alternative to large-scale farming, micro eco-farmers may better satisfy the needs of those consumers who are driven by anti-mass production sentiments. This would represent a re-partitioning of the farm produce market, with micro eco-farmers representing the true specialist form identity.

Finally, it is interesting to note that the micro eco-farming movement places itself in opposition not only to large-scale farming techniques but also to official organic certification programs. This seems to indicate a specialist form identity that could meet the needs of consumers seeking true "alternatives" to conventional produce, and whose purchasing decisions express a distinct, non-inclusive identity. Whether this form will eventually become established and institutionalized remains to be seen but, if successful, it should appeal to a broad but homogeneous base of strongly identifying adherents. The impression that we obtained from our interaction with micro eco-farming movement members is that - just as in the case of micro-brewers and micro-radio activists - their community resembles a tight-knit network of followers. When looking for our case data and contacting movement participants, we were readily directed from one movement 
organization to the other. Micro eco-movement members know each other; they know who their enemy is; and they know what their identity is about, which may facilitate the detection and sanctioning of violations of their organizational form identity necessary to sustain market partitioning. Through appropriate framing of movement goals, articulation of an oppositional strategy, and appeal to a targeted base and infrastructure, this movement may succeed in stably partitioning the market in a way that earlier organic farmers failed to accomplish.

\section{Discussion}

Our analysis offers three important contributions to extant research at the intersection of social movements and resource partitioning theory. First, we emphasize the role of movement dynamics in resource partitioning. Although shared goals, adept framing and discourse, and a supportive social and community infrastructure may indeed be fruitful drivers of market partitioning, they change as movements unfold, grow larger, and become more successful. As a result, by influencing the formation and nature of specialist form identities movement dynamics may trigger processes of de- and re-partitioning.

Second, we argue that because participants' identification with movement goals weakens as movements expand to attract more diverse adherents, the definition of the specialist form identity becomes increasingly important for sustaining market partitioning over time. Because movement participants' identification is critical to sustaining loyalty to the common cause and the investigation, detection and sanctioning of violations of the specialist form identity, those movements best able to grow their bases may ultimately sacrifice their strength. When movement participants do not recognize and sanction identity code violations, generalists are free to enter the specialist resource space, engendering direct competition between the two forms. Only by taking a dynamic view of identity movements does this tension become apparent.

Finally, we suggest that specialist form identities that incorporate limits to organizational growth are more viable than those that do not. We point to the rise of micro eco-farms, a new specialist form identity that satisfies this condition and may be more likely to sustain itself as a viable alternative to conventional farming as an important turning point in the broader organic movement. We conclude that this newly emerging, sharply defined specialist form identity may successfully induce a re-partitioning of the farming produce market in the current stage of the movement.

Limits to organizational growth have direct implications for the specification of the original resource partitioning model. Formal statements of the original theory maintain that, as the number of dimensions in the resource space increases, the total amount of space open to specialists expands which, in turn, leads to both higher founding and lower mortality rates of specialist organizations (Carroll et al., 2002). Given our conclusions regarding the limits to organizational growth for the specialist form, the specialist founding and mortality hypotheses must be refined. That is, we put forward limits to organizational growth as a necessary condition for movement-based resource partitioning and conjecture that, as the space open to specialists expands, there will be an increase in the viability of those specialist organizations whose form identity embodies limits to organizational growth. Moreover, when limitations to growth are present in the organizational form identity, increased demand for specialist products can only be met by an increase in specialist density, but not by the growth in scale of a few specialist organizations. Without such limits, generalists may again become capable of entering the specialist resource space and competing directly with specialists, ultimately crowding them out. 


\section{Theoretical and Practical Implications}

Our study has a number of important implications. First, the crowding out of specialist organizations in the event of de-partitioning may lead to excessive mortality rates of specialist organizations. The USDA statistics seems to confirm this assumption: as its statistics show, since the 1960s the number of farms has declined, although the average acreage per farm has increased (Dimitri, Effland, \& Conklin, 2005).

Second, the fact that movement-based partitioning, regardless of size or success, may engender processes of re- and de-partitioning should be reassuring for movement adherents, producers and consumers, who spend significant time and resources fighting for their cause. Although the organic movement did result in significant, broad-based changes to farming practices and although as consumers we are able to purchase more organic fruits and vegetables at a more reasonable price, market de-partitioning puts organic farmers at an ideological crossroads: remain small, and find new bases for differentiation by remaining authentic and true to the movement's ideals, or resort to identity management, minimally adhering to both their core values and standards and competing directly with conventional farms. One problem associated with this outcome is that, if organic farms are only able to survive by abandoning some of their ideals and growing, these ideals may soon be lost. Another problem is that identity management may go beyond pretending that the produce comes from a small self-sufficient farmstead; rather, some farmers reap a considerable price premium for produce that does not meet consumers' expectations or that may be fraudulently labeled as organic. Consumer and other watchdog organizations have documented several incidents of produce being fraudulently represented as organic. ${ }^{1}$

Our research also points to the importance of defining the specialist form identity in enhancing specialist viability and sustaining market partitioning. Identity movement leaders who push for change, therefore, should be wary of emerging specialist form identities that are too broad or too close to generalist form identities to be uniquely identifiable. Instead, they are well advised to push for standards representing narrow and sharply defined form identities that are unequivocally distinct from generalists. Moreover, in comparing the organizational form identity of micro-breweries, micro-radio broadcasters, and micro eco-farms, we suggest that specialist form identities that incorporate limitations to organizational growth may be more viable than those that do not.

Even if limitations to growth may be a fruitful avenue for specialists to explore, however, there remains the important issue of organizational diversity. That is, if only very small organizations are able to proliferate and survive in concentrated markets, then the meaningfulness of organizational diversity in the face of generalist domination is questionable. In the production of cultural goods like movies or print media, for example, where identity movements most frequently arise, preserving an open marketplace for ideas is indispensable for the functioning of democratic institutions. It follows that if specialists reach only small audiences because of their very small size and limited purview, this may have significant implications for society at large. Thus, despite its merits for re-partitioning, there may be a social cost to the incorporation of limits to size in specialist form identity.

\section{Alternative Explanations}

Although we kept an eye to evidence disconfirming our findings as we conducted our analysis, several alternative explanations merit particular attention. The primary alternative to our argument may be that the farming resource space may not lend itself to partitioning. In his canonical work on resource partitioning, Carroll (1985, p. 1272) enumerates seven assumptions underlying market 
partitioning. Having considered each extensively, we conclude they are satisfied by the organic farming movement and feel justified in arguing that organic farming does, in fact, lend itself to market partitioning.

Another possible explanation is that we have improperly characterized the stages of movement and market development in building our case. That is, the movement never generated specialist organizations that served small, specialized niches and the growth of organic farming proceeded in as straightforward a manner as, for example, the use of poison pills or hybrid corn (e.g., Strang \& Soule, 1998). Although one could argue that the popularity of organic produce has followed a traditional pattern of diffusion fueled by social movements, our reading of the organics story reveals a more nuanced interaction between movement and market; that is, as the movement progresses, it impacts the structure of competition itself. Our conversations with movement participants and scholars, as well as our engagement with the extant literature on the organic farming movement, all support our characterization of the market's development and its current state.

It is also possible that the current state of the organic market is entirely an artifact of the regulatory process. Standards, be they government- or self-imposed, are critical in protecting and institutionalizing specialist form identity; thus, it is possible that the certification process alone, not the organic farming movement, is responsible for the erosion of boundaries between generalists and specialists. We have considered this possibility at length, and suggest that, while the national organic standard indeed allows for a very broad definition of the organic farming, it is the outcome - not the cause - of market de-partitioning. The broad formulation of current US standards stems directly from the mechanisms underlying the organic movement. Prior research shows that the definition of the specialist form identity by movement participants precedes its institutionalization by formal institutions, such as government agencies and regulators (Carroll \& Swaminathan, 2000; Greve et al., 2006). In identity movements such as micro-radio and micro-brews, where movement participants united around shared goals and an unequivocally identified common enemy, specialists were able to portray their form as the solution to generalist, homogenous domination, then bridge the gap to institutions that standardized those form identities. In the organic case, however, the lack of shared goals and the conflicting motives of agrarians and expansionists prevented both the establishment of a sharply defined organic form identity and its formal institutionalization. Even though both camps reached out for support from trained organization builders and pushed for the creation of standards, the contradictory motives within the organic camp prevented such standardization on the federal level. This spurred a de-partitioning of the market for farming products and allowed generalist organizations and those adopting more conventional farming methods to invade specialists' resource space. That the generalists were able to push for flexible and poorly enforced standards is thus a secondary effect, the consequence rather than the cause of a poorly defined organizational form identity.

\section{Limitations and Conclusions}

As all research, our study suffers from several limitations. First, although we have tapped a wealth of rich qualitative data, the limited availability of quantitative data on the structure of the organic farming industry precluded method triangulation: to corroborate our findings further, we initially strove to analyze our structured data through quantitative methods. We attempted to gather data on our own by contacting regulators, certifiers, social organizations, and academics who specialize in organic farming, but were unable to overcome this obstacle. In our qualitative review, however, we 
found sufficient consensus about the growth and progress of the organic movement and industry that our presentation of the case is as accurate as it can be.

In addition, given that the term organic farming encompasses a number of very different farm products, including fruits, grains, and vegetables, another limitation of our study may be that we did not focus on a single product offering with more definable characteristics, such as organic dairy products or heirloom tomatoes. Admittedly, farm count and farm structure may differ across categories of produce, as does government regulation. Our choice was motivated by fact that the salient issue in our thinking is the method of production, rather than the product itself. In fact, as in similar cases at the intersection of identity movements and resource partitioning, it is the specialists' method of production rather than product characteristics that lead to market partitioning. As Carroll and Swaminathan (2000) note, micro-brew aficionados' buying decisions are driven not merely by quality, but also by the identity of the producer and its adherence to production methods considered to be authentic. Choosing organic farming broadly allows us to focus on broad production standards related to a broad movement, rather than techniques specific to particular farm products or characteristics thereof.

Future research might use quantitative techniques to test our arguments. While there are many important issues in this domain that could be fruitfully explored, we would like to highlight the role of individual and social identity in driving organizational founding. Although organizational ecologists recognize the role of identity movements in market structure, they have largely overlooked the role of movement participants' own identities in the process of specialist founding and viability. That is, existing research tends to conceptualize identity movements through the consumer, whereas producers are equally likely to belong and respond to identity movements. This is important, because if producers themselves are movement adherents, their product offerings may precede demand for their goods, in contrast to the traditional view that specialist foundings respond to already demonstrated market demand. Similarly, changes to movement participants' identification over time bear important implications for specialist viability.

Finally, as movements grow and become more heterogeneous, some adherents may splinter off into smaller groups, seeking balance between inclusion and distinctiveness (Brewer, 1991). Although not part of our original argument, this striving for optimal distinctiveness might have been one of the mechanisms that led micro eco-farmers to break away from the organic movement, develop a new specialist form identity, and engender a re-partitioning of the market for farm products. Thus future research might address how changes to producer identification with identity movements can lead to market re-partitioning.

This study uses a case that might have led to market partitioning to explore resource partitioning theory in a nuanced way. By bringing in the dynamics of identity movements, we have sought to understand the factors that led the organic farming industry to become de-partitioned over time, and which may well lead to re-partitioning in the near future. In addition, by comparing and contrasting our case to those in which the predictions of resource partitioning were borne out, we were able to refine the ecological argument by adding an important scope condition. This research is the first step in the development of a more dynamic view of resource partitioning theory.

\section{Acknowledgements}

We would like to thank our editors and reviewers, Heather Haveman, Brandon Lee, Michael Lounsbury, Sanaz Mobasseri, Amy Kim, Samantha Johnston, Lisa Xue, Kelly Yang, Matthew Barker-Benfield, and participants in the "Modern Agricultural Processes: There and Back Again" symposium at the 2009 Academy of Management meetings in Chicago. An early version of this study was nominated for the 2008 Best Paper for Practice Implications Award by the Strategic Management Society. 


\section{Funding}

This research received no specific grant from any funding agency in the public, commercial or not-for-profit sectors.

\section{Note}

1. See, for example, http://www.organicconsumers.org/sos.cfm.

\section{References}

Adams, B. B. (2004). Micro eco-farming: prospering from backyard to small acreage in partnership with the earth. Auburn, CA: New World Publishing.

Barnett, E. (2011). Personal interview by author with Erin Barnett, Director of Local Harvest, 23 September.

Bartley, T. (2003). Certifying forests and factories: States, social movements, and the rise of private regulation in the apparel and forest products fields. Politics \& Society, 31, 433-464.

Benford, R. D., \& Snow, D. A. (2000). Framing processes and social movements: An overview and assessment. Annual Review of Sociology, 26, 611-639.

Brewer, M. B. (1991). The social self: On being the same and different at the same time. Personality and Social Psychology Bulletin, 17, 475-482.

Brewers Association (2011). Brewers Association: A passionate voice for craft brewers. http://www.brewersassociation.org.

Buck, D., Getz, C., \& Guthman, J. (1997). From farm to table: The organic vegetable commodity chain of northern California. Sociologia Ruralis, 37(1), 3-20. doi: 10.1111/1467-9523.00033.

Buechler, S. M. (1995). New social movement theories. Sociological Quarterly, 36, 441-464.

Carroll, G. R. (1985). Concentration and specialization: Dynamics of niche width in populations of organizations. American Journal of Sociology, 90, 1262-1283.

Carroll, G. R., Dobrev, S., \& Swaminathan, A. (2002). Organizational processes of resource partitioning. Research in Organizational Behavior, 24, 1-40.

Carroll, G. R., \& Swaminathan, A. (2000). Why the microbrewery movement? Organizational dynamics of resource partitioning in the US brewing industry. American Journal of Sociology, 106, 715-762.

Center for the Micro Eco-Farming Movement. (2010). http://www.microecofarming.com.

Concord, P. (2001). The origin of the organic movement. Edinburgh, UK: Floris Books.

Dimitri, C., Effland, A., \& Conklin, N. (2005). The transformation of US agriculture and farm policy. Economic Information Bulletin, Number Three. http://www.ers.usda.gov.

Foreman, P., \& Whetten, D. A. (2002). Members' identification with multiple-identity organizations. Organization Science, 13, 618-635.

Fromartz, S. (2006). Organic, Inc.: natural foods and how they grew. Orlando, FL: Harcourt Books.

Greene, C. (2001). Organic labeling. In E. Golan, F. Kuchler, L. Mitchell, C. Greene, \& A. Jessup (Eds.), Economics of Food Labeling (pp. 26-29). Washington, DC: US Department of Agriculture.

Greve, H. R., Pozner, J.-E., \& Rao, H. (2006). Vox populi: Resource partitioning, organizational proliferation, and the cultural impact of the insurgent microradio movement. American Journal of Sociology, 112, 802-837.

Guthman, J. (2000). Raising organic: An agro-ecological assessment of grower practices in california. Agriculture and Human Values, 17, 257-266.

Guthman, J. (2004). Agrarian dreams: the paradox of organic farming in California. Berkeley: University of California Press.

Hannan, M. T. (1991). Theoretical and methodological issues in analysis of density-dependent legitimation in organizational evolution. Sociological Methodology, 21, 1-42.

Heckman, J. (2006). A history of organic farming: Transitions from Sir Albert Howard's war in the soil to USDA national organic program. Renewable Agriculture and Food Systems, 21, 143-150.

Ingelhart, R. (1990). Values, ideologies and cognitive mobilization in new social movements. In R. J. Dalton, \& M. Kuechler (Eds.), Challenging the political order: new social and political movements in western democracies (pp. 43-66). New York, NY: Oxford University Press. 
Jasper, J. M. (1997). The art of moral protest. Chicago, IL: University of Chicago Press.

King, M. (2007). Organic labeling confuses farmers. Times-Herald Record, 11 November. http://www. recordonline.com

Klonsky, K., Tourte, L., Kozloff, R., \& Shouse, B. (2002). A statistical picture of California's organic agriculture 1995-1998. DANR Publication no. 3425. University of California Agricultural Issues Center.

Lee, B., Sine, W. D., \& Tolbert, P. (2011). Certifying the harvest: the role of standards-based certification organizations in the organic food industry. London: London Business School.

Lohr, L., \& Park, T. (2002). Choice of insect management portfolios by organic farmers: Lessons and comparative analysis. Ecological Economics. http://www.sciencedirect.com/science.

Lounsbury, M., Ventresca, M., \& Hirsch, P. M. (2003). Social movements, field frames and industry emergence: A cultural-political perspective on U.S. recycling. Socio-Economic Review, 1(1), 71-104.

McAdam, D. (1999). Political process and the development of Black insurgency, 1930-1970. Chicago, IL: University of Chicago Press.

McKendrick, D. G., \& Carroll, G. R. (2001). On the genesis of organizational forms: Evidence from the market for disk arrays. Organization Science, 12, 661-682.

Meyer, D. S., \& Whittier, N. (1994). Social movement spillover. Social Problems, 41, 277-298.

Mezias, J. M., \& Mezias, S. J. (2000). Resource partitioning, the founding of specialist firms, and innovation: The American feature film industry, 1912-1929. Organization Science, 11, 306-322.

Natural Marketing Institute, The (2005). Organic food \& beverage sales increase 18\%; Household penetration decreases. http://www.npicenter.com/anm/templates/newsATemp.aspx?articleid=11772\&zoneid=6 (accessed 26 June 2008).

Ness, C. (2006). Green Giants: Mega-producers tip scales as organic goes mainstream. San Francisco Chronicle. http://www.sfgate.com.

Nestle, M. (2007). What to eat. New York, NY: North Point Press.

Oberschall, A. (1973). Social conflict and social movements. Englewood Cliffs, NJ: Prentice-Hall.

Peterson, R. A. (1997). Creating country music: fabricating authenticity. Chicago, IL: University of Chicago Press.

Pichardo, N. A. (1997). New social movements: A critical review. Annual Review of Sociology, 23, 411-430.

Pollan, M. (2006). The omnivore's dilemma : a natural history of four meals. New York, NY: Penguin Press.

Polletta, F., \& Jasper, J. M. (2001). Collective identity and social movements. Annual Review of Sociology, 27, 283-305.

Polos, L., Hannan, M. T., \& Carroll, G. R. (2002). Foundations of a theory of social forms. Industrial and Corporate Change, 11, 85-115.

Pozner, J. E., \& Rao, H. (2006). Fighting a common foe: Enmity, identity and collective strategy. In J. A. C. Baum, D. S. Dobrev, \& A. van Witteloostuijn (Eds.), Ecology and Strategy (Vol. 23, pp. 445-479). Amsterdam: Elsevier Ltd.

Ragin, C. C. (1994). Constructing social research: the unity and diversity of method. Thousand Oaks, CA: Pine Forge Press.

Rao, H. (1994). The social construction of reputation: Certification contests, legitimation, and the survival of organizations in the American automobile industry 1895-1912. Strategic Management Journal, 15, 29-44.

Sligh, M., \& Christman, C. (2003). Who owns organic? The global status, prospects, and challenges of a changing organic market. Pittsboro, NC: Rural Advancement Foundation International.

Soule, S. A., \& King, B. G. (2008). Competition and resource partitioning in three social movement industries. American Journal of Sociology, 113, 1568-1610.

Strang, D., \& Soule, S. A. (1998). Diffusion in organizations and social movements: From hybrid corn to poison pills. Annual Review of Sociology, 24, 265-290.

Agriculture Research and Education (2008). Cooperative marketing/campaigns. SARE Bulletin. http://www. sare.org/publications/marketing/market08.htm.

Vos, T. (2000). Visions of the middle landscape: Organic farming and the politics of nature. Agriculture and Human Values, 17, 245-256. 
Walz, E. (2004). Fourth national organic farmers' survey. Organic Farming Research Foundation. http://ofrf. org/publications/pubs/4thsurvey_results.pdf.

Weber, K., Heinze, K., \& DeSoucey, M. (2008). Forage for thought: Mobilizing codes in the movement for grass-fed meat and dairy products. Administrative Science Quarterly, 53, 529-567.

Yin, R. K. (1984). Case study research: design and methods. Beverly Hills, CA: SAGE Publications.

Yin, R. K. (1998). The abridged version of case study research: Design and method. In L. Bickman \& D. J. Rog (Eds.), Handbook of applied social research methods (pp. 229-260). Thousand Oaks, CA: SAGE Publications.

\section{Author biographies}

Katarina Sikavica is a post-doctoral researcher at the Institute of Strategic Management at the Ludwig Maximilians University Munich, Germany. Her research interest centers on new forms of collective action by organizational stakeholders and the implications thereof from the point of view of corporate strategy and governance. In her more recent work she focuses on psychological foundations of shareholder activism and the concept of organizational ownership.

Jo-Ellen Pozner is an Assistant Professor of Management of Organizations at the Haas School of Business at the University of California, Berkeley. Her research focuses on questions of organizational legitimacy, with particular emphasis on organizational misconduct, impression management, and corporate governance. Her recent work deals with the social, psychological and sociological forces that led to the subprime mortgage crisis. 